\title{
Structural changes in renal arterioles in Type I diabetic patients
}

\author{
R. Østerby ${ }^{1}$, A.Hartmann ${ }^{3}$, H.-J. Bangstad ${ }^{2}$ \\ ${ }^{1}$ Electron Microscopy Laboratory, Aarhus Kommunehospital, Aarhus, Denmark \\ ${ }^{2}$ Aker and Ullevål Diabetes Research Center, Oslo, Norway \\ ${ }^{3}$ Medical Department B, Rikshospitalet, Oslo, Norway
}

\section{Abstract}

Aims/hypothesis. We aimed to obtain data on arteriolar structure in a follow-up study of microalbuminuric diabetic patients.

Methods. Kidney biopsies were obtained at baseline and after 8 years in 18 Type I (insulin-dependent) diabetic patients. Albumin excretion rate, blood pressure and $\mathrm{HbA}_{1 \mathrm{C}}$ were measured regularly, and the glomerular filtration rate (GFR) was measured at the time of the kidney biopsy. The biopsy was embedded into plastic blocks and serially sectioned with $1 \mu \mathrm{m}$ sections. In levels $25 \mu \mathrm{m}$ apart, afferent and efferent arteriolar profiles were identified and digitised in the electron microscope. The extra-cellular matrix as volume fraction of the media was measured, and estimates of thickness of matrix, media, endothelium and lumen were obtained. Baseline and follow-up biopsies were studied concomitantly.

Results. A large increase was seen in matrix volume fraction in afferent $(p=0.0001)$ and in efferent arterioles $(p=0.0004)$. Estimated thickness of media and matrix increased, whereas endothelial cell thickness decreased, over the 8 years. There was a correlation between the parameters of diabetic glomerulopathy and arteriolar parameters in the biopsies done at 8 years, basement membrane thickness compared with afferent matrix volume fraction: $r=0.74$, $p=0.0005$. Also aggravation of glomerulopathy and arteriolar structure over 8 years showed positive correlation. Arteriolar parameters correlated with the albumin excretion rate (AER) and inversely with GFR.

Conclusion/interpretation. The arteriolar accumulation of matrix parallels that taking place in glomeruli and shows association with functional parameters over 8 years in Type I diabetic patients with microalbuminuria. These changes are considered an important part of the structural lesions in the diabetic kidney underlying the development of diabetic nephropathy. [Diabetologia (2002) 45:542-549]

Keywords Arteriolar hyalinosis, diabetic glomerulopathy, diabetic nephropathy, microalbuminuria, renal arterioles, renal biopsy, stereology, Type I diabetes.
Abnormalities of arteriolar structure in the diabetic kidney were described in histological studies in the

Received: 9 October 2001 and in revised form: 29 November 2001

Corresponding author: R. Østerby, Electron Microscopy Laboratory, Building 3, Aarhus Kommunehospital, Aarhus C., Denmark, e-mail: ruth.osterby@dadlnet.dk

Abbreviations: $\mathrm{V}_{\mathrm{V}}$ (matrix/media) Volume fraction of matrix in arteriolar media; Matrix T estimated matrix thickness ( = volume/surface-ratio); GFR, glomerular filtration rate; AER, albumin excretion rate; JGA, juxtaglomerular 1940's and 1950's (reviewed in [1]). The lesion consisting of a homogeneous material in the vessel wall was termed arteriolar hyalinosis due to the appearance of the PAS-positive a-cellular material. Staining characteristics were similar to those of glomerular fibrinoid caps and capsular drops. All these changes were therefore referred to one group, the exudative lesions [2], and increased leakage of plasma protein into the arteriolar wall was considered an important pathogenetic mechanism [3].

Arteriolar hyalinosis is also observed in the kidneys of elderly non-diabetic patients and in hyperten- 
sion $[4,5]$. But, the frequent affection of the efferent as well as the afferent arteriole in the diabetic kidney seems to be at variance with non-diabetic cases.

When present, the arteriolar hyalinosis is distributed over the entire cortex of the kidney, but within narrow windows it is focal: Along the course of one arteriole, segments can be maximally abnormal, whereas others appear with a normal structure, and that individual arteriolar profiles in the sections can have a wide range of appearances.

In advanced stages the arteriolopathy is an eyecatching part of the structural abnormalities in the diabetic kidney but the lesion can be present fairly early. In kidneys transplanted into diabetic recipients, arteriolar hyalinosis was reported to be present within the first 5 years [6]. Frequent occurrence of arteriolar hyalinisation, evaluated qualitatively, was reported in young patients with a short duration of diabetes [7]. In a study using quantitative electron microscopy in the early stage of microalbuminuria the arteriolar structure was found to be significantly different from that in non-diabetic control subjects as well as from that in diabetic patients with normoalbuminuria [8]. The lesion therefore probably develops independently of increases in systemic blood pressure [9] and it could be considered an expression of the metabolically determined accumulation of extra-cellular material in analogy with that in diabetic glomerulopathy.

One follow-up study of renal arteriolar structure in Type I diabetic patients with microalbuminuria covering a 3-year period has been published, in which the patients received antihypertensive treatment [10]. An increase in the extra-cellular matrix was observed over this period of time in the afferent arterioles in the group of patients treated with beta-blockers, whereas the group treated with ACE inhibitor did not have a significant increase.

In this study, we followed the renal structural changes over a period of 8 years in young Type I diabetic patients, who were initially microalbuminuric. These patients did not receive antihypertensive treatment except for one case.

\section{Subjects and methods}

The patients. A total of 18 Type I diabetic patients are included in the present follow-up study. The inclusion criteria were described in a previous report in detail [11]. The series included diabetic patients over 18 years of age, diabetes duration of more than 5 years and with persistent microalbuminuria at the time of the baseline biopsy. Microalbuminuria was defined as albumin excretion rate (AER) between $15-200 \mu \mathrm{g} / \mathrm{min}$ in at least two of three consecutive overnight urine samples. After a baseline renal biopsy had been taken, the patients entered a clinical follow-up study over the following $2.5-3$ years, being allocated to either treatment with insulin pumps or conventional treatment with multiple daily injections of insulin. Clini-
Table 1. Clinical data in the series at entry into the study, at the time of the 8-years biopsies or during follow-up. Means and (SD), for AER median and (range)

\begin{tabular}{lcc}
\hline & Baseline & Follow-up \\
\hline Age & $20(3.6)$ & $28.3(3.4)$ \\
Duration of diabetes & $11(2.9)$ & $19.4(2.9)$ \\
AER $(\mu \mathrm{g} / \mathrm{min})$ & $32.5(15-194)$ & $17.5(2-744)$ \\
GFR $\left(\mathrm{ml} \cdot \mathrm{min} \cdot 1.73 \mathrm{~m}^{2}\right)$ & $140(27.2)$ & $125(20.3)$ \\
Systolic blood pressure $(\mathrm{mmHg})$ & $128(11.5)$ & $126(9.4)^{\mathrm{a}}$ \\
Diastolic blood pressure $(\mathrm{mmHg})$ & $80(8.5)$ & $77.6(6.6)^{\mathrm{a}}$ \\
HbA $_{1 \mathrm{C}}(\%)$ & $10(1.4)$ & $9.3(1.3)^{\mathrm{a}}$ \\
\hline
\end{tabular}

${ }^{\text {a} M e a n s ~ d u r i n g ~ 8-y e a r s ~ f o l l o w-u p ~}$

cal data in the group of patients at the time of baseline biopsy is shown in Table 1.

The controlled clinical trial lasted for an average of 30 months (range 24-33). At this time a second biopsy was taken, and hereafter the strict treatment protocol was not adhered to. But all of the patients were followed regularly with clinical examinations: clinical blood pressure, AER and $\mathrm{HbA}_{1 \mathrm{C}}$ every fourth month. GFR was measured at the time at which the kidney biopsies were taken. A third biopsy was carried out after an average of 99 months (range 84-104). Two patients in the original cohort dropped out due to pregnancy. Another two patients did not follow the treatment protocol in the prospective study but were followed clinically and had the 8-years biopsy taken. They are included in this study, which deals with data from the baseline biopsies and from the third biopsies, which are, in the following, termed the 8years biopsies.

This study was approved by the local ethics committee and all patients gave their written consent.

Renal biopsies. Percutaneous renal biopsies were taken under ultrasound guidance using an 18-gauge needle. The biopsy specimen was fixed in $2 \%$ buffered glutaraldehyde and mailed in the fixative to Aarhus. The core was subdivided into smaller blocks which were dehydrated and embedded. As embedding medium vestopal was used for the baseline biopsies and epon for the 8-years biopsies.

Sampling for electron microscopy. Each of the plastic embedded blocks was serially cut with $1 \mu \mathrm{m}$ thick sections. All sections were picked up on slides and stained with toluidine blue. Levels with $25 \mu \mathrm{m}$ intervals were used to trace any arteriolar profiles on the section. If afferent or efferent arterioles were present in the sections, thin sections for electron microscopy were cut. When the identification of profiles as afferent or efferent arterioles was uncertain, serial sections were followed to trace the further course of the arterioles for correct identification. Furthermore, a limit was set for the efferent arterioles which gradually transform from arterioles into capillary structures, where only profiles within the first $100 \mu \mathrm{m}$ from the vascular pole were included. The exact determination of distance from the vascular pole has been described in detail previously [10]. In short, the distance in the serial sections from exit in the vascular pole to the current level was calculated from horizontal and vertical distances using Pythagoras' theorem. The distance between levels was estimated by measuring in each block at the start of sectioning the distance from the section surface to a parallel groove cut on the side of the block. The measurement consisted in counting cycles of $1 \mu \mathrm{m}$ with the ultra-microtome. After sectioning through the block the distance between section plane and the groove was re-measured. With 


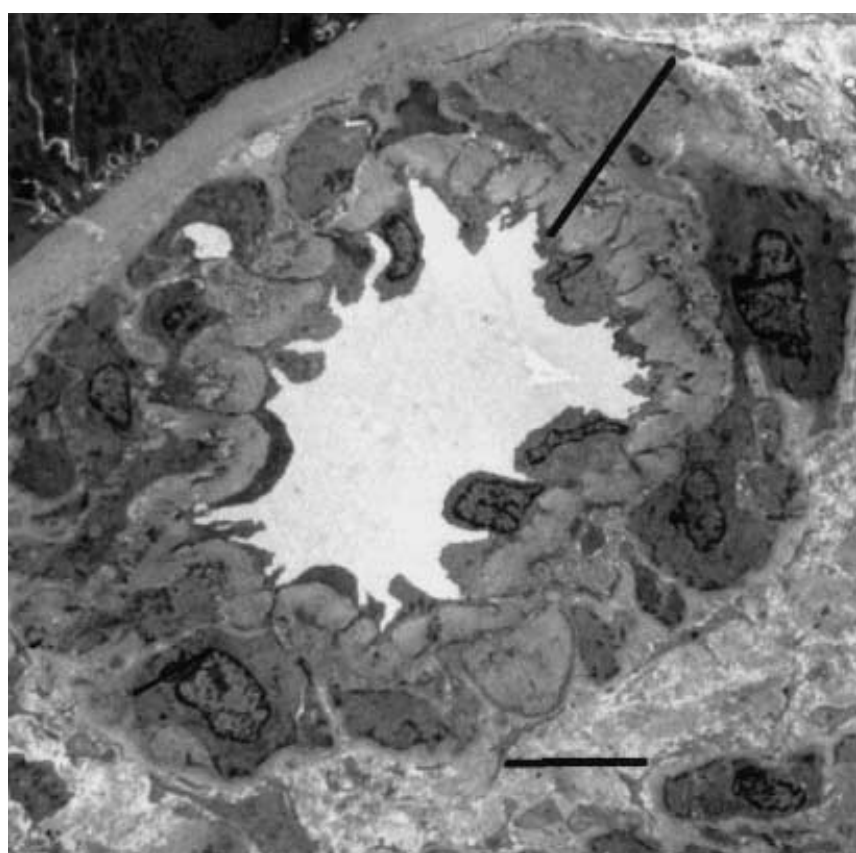

Fig. 1. Profile of afferent arteriole. Media: From base of endothelial cell to periphery, indicated by a line. The arteriolar matrix is identified as all extra-cellular material in media. Electron micrograph. The bar is $5 \mu \mathrm{m}$

the known number of levels in between, the average distance between levels was then calculated.

The thin sections were cut from untrimmed blocks providing a large area for sampling of arterioles.

Electron microscopy. Any arteriolar profile present on the section (efferent if within $100 \mu \mathrm{m}$ from the vascular pole) was recorded at the electron microscope. For the present study all images were digitally recorded by the SIS system (AnalySIS 3.0, Soft Imaging System, Münster, Germany) connected to the Philips CM10 microscope (Eindhoven, The Netherlands).

All baseline biopsies were recorded and measured together with the 8-years biopsies.

Using a light microscope with a drawing tube, sketches of the semi-thin sections were made with each arteriolar profile indicated. The profiles were then located in the electron microscope and recordings were obtained which covered completely the full arteriolar profile. Images were stored on a disk, which was taken to the office computer for measurements.

Measurements. With each image transferred to the computer screen a grid designed for the present purpose was also transferred to the screen using a grid programme (DIMAC, Digital Image Company, CHAMP, Cytology and History Analysis Modular Package, Arhus, Denmark). An integral grid with coarse and fine points, in a ratio of 2 to 1 was used. The distance between coarse points was $4 \mu \mathrm{m}$. Coarse points were used for hits on arteriolar media (Figs.1-2), fine points for hits on any extra-cellular material (matrix), on matrix with a fluffy or electron dense appearance, endothelium and lumen. Grid lines with a length of $8 \mu \mathrm{m}$ for each coarse point were used for counting of intersections with the interface between the circumference of the profile towards the interstitium and the interface between the endothelial cell and media. The magnification on the computer screen was $\times 9300$.

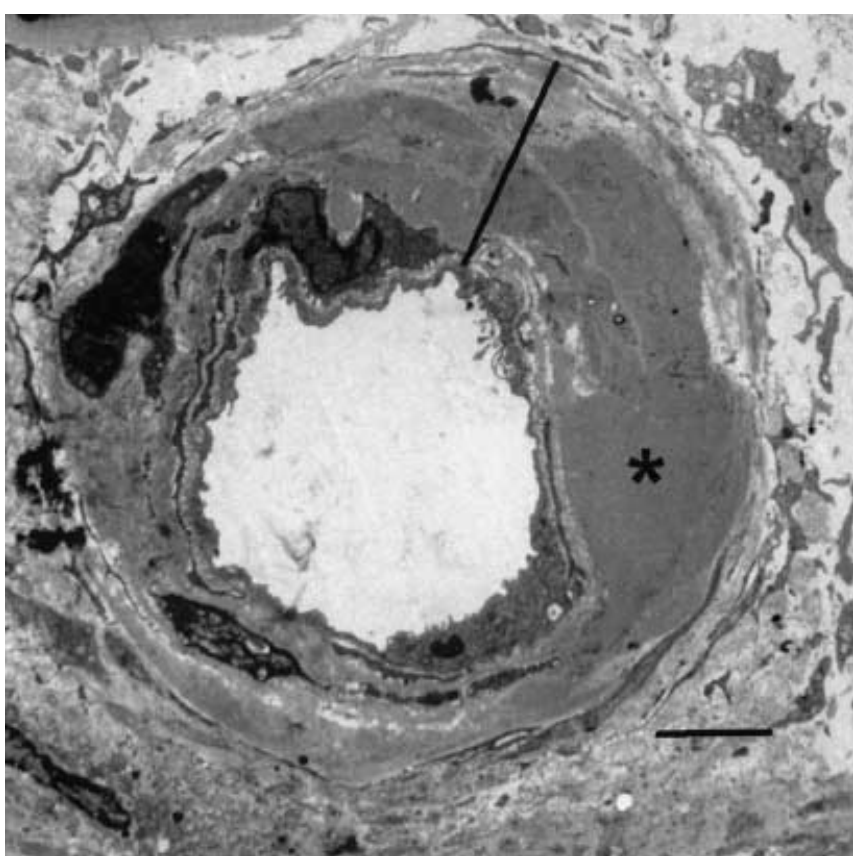

Fig. 2. Electron micrograph showing a profile of an efferent arteriole located $10 \mu \mathrm{m}$ from the vascular pole. At this location the matrix constitutes a large fraction of total media and contains electron dense areas $(*)$. The arteriolar media is indicated by a line. The bar is $5 \mu \mathrm{m}$

Calculations. The point counting provided the estimates of volume fraction of matrix using total arteriolar media as the reference space, $\mathrm{V}_{\mathrm{V}}$ (matrix/media). Media is defined as the space between the peripheral endothelial cell membrane and the interstitium, as indicated in Figures 1-2. An arbitrary thickness $\mathrm{T}$, was calculated as the volume-to-surface ratio $[8,10]$ for total media, matrix, endothelium and lumen. The luminal $\mathrm{T}$ corresponds to luminal radius.

An average of 15 afferent profiles (range 8-22) and 13 efferent profiles (range 8-18) were measured for each baseline biopsy. For the 8-years biopsies the corresponding figures were 18 (11-35) for afferent, and 17 (11-22) for efferent arterioles.

In the measurements, it was recorded whether or not the arteriolar profile was attached to the vascular pole in the plane of sectioning. Only unattached profiles were used for the estimates of the calculated thickness T.

Statistics. Comparisons between baseline and follow-up biopsies were done with Student's two-sided paired $t$ test. Correlations were tested with least squares regression. A $p$ value of less than 0.05 was considered to be statistically significant.

\section{Results}

The clinical course during the follow-up period. Clinical data recorded during the entire follow-up period or at the time of the 8-years biopsies are shown in Table 1 .

At the termination of the project, five of the patients were normoalbuminuric and two had AER of 


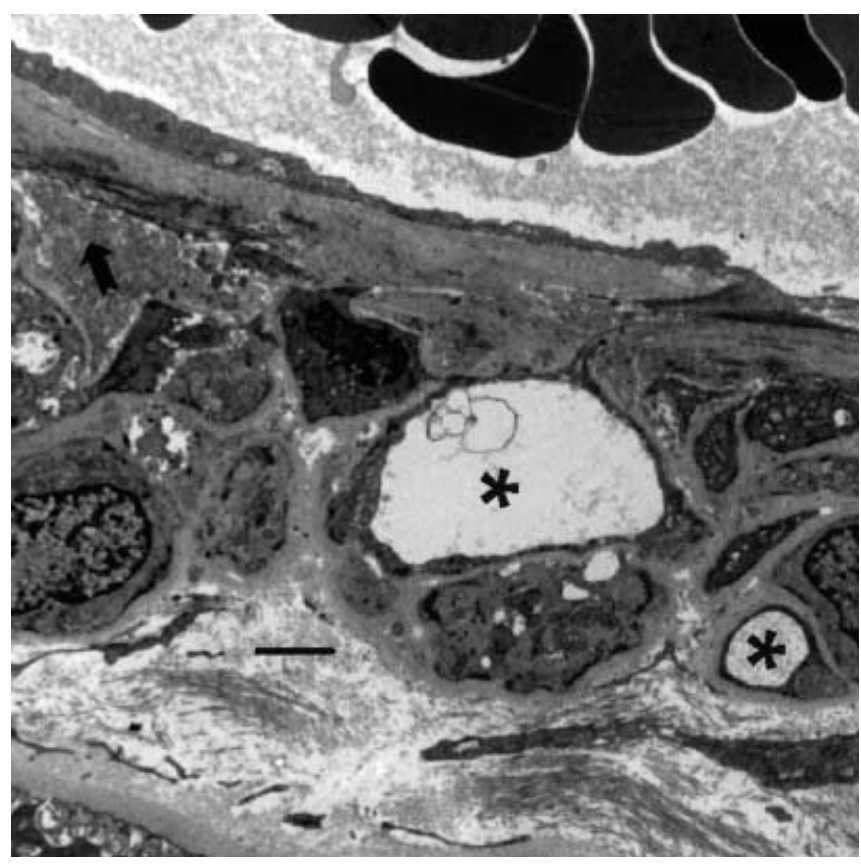

Fig. 3. Section of the wall of an afferent arteriole. Large vacuoles are seen in a couple of myomedial cells $(*)$, and an area with membranous structures is present in the matrix (arrow). The bar is $2 \mu \mathrm{m}$

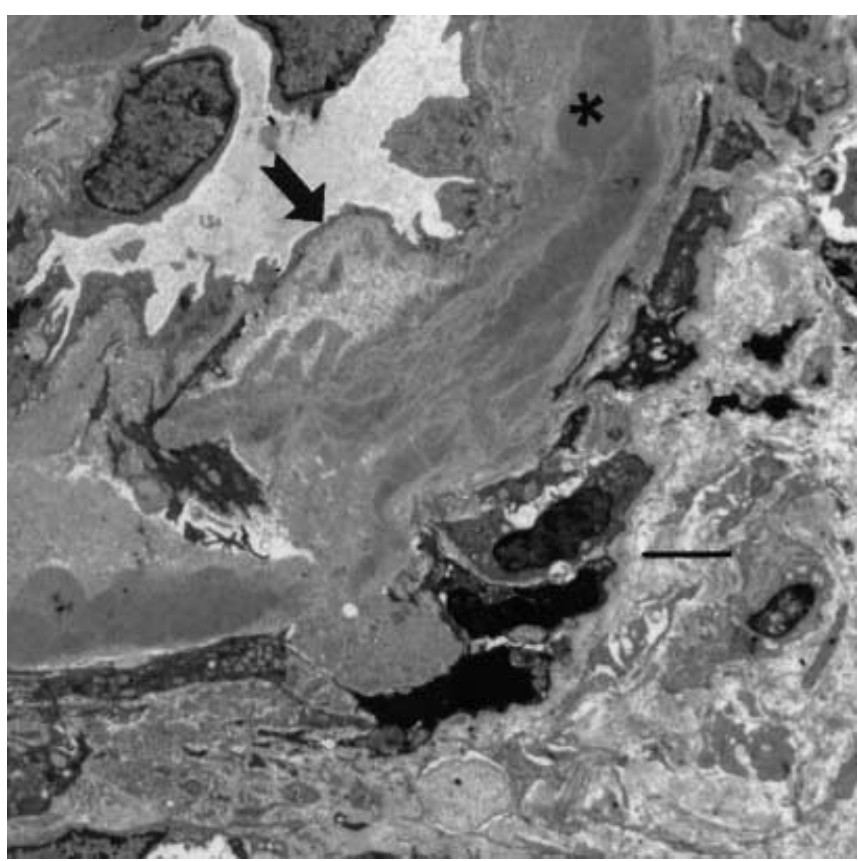

Fig. 4. In the matrix of this afferent arteriole fluffy (arrow) and electron dense areas (*) are seen. The bar is $3 \mu \mathrm{m}$

over $200 \mu \mathrm{g} / \mathrm{min}$, the rest of the series remained microalbuminuric. For the whole group there was a reduction in the GFR $(p=0.05)$. The clinical blood pressure did not show any change. One patient developed hypertension and was treated with ACE-inhibitor during the last 2 years of the study.

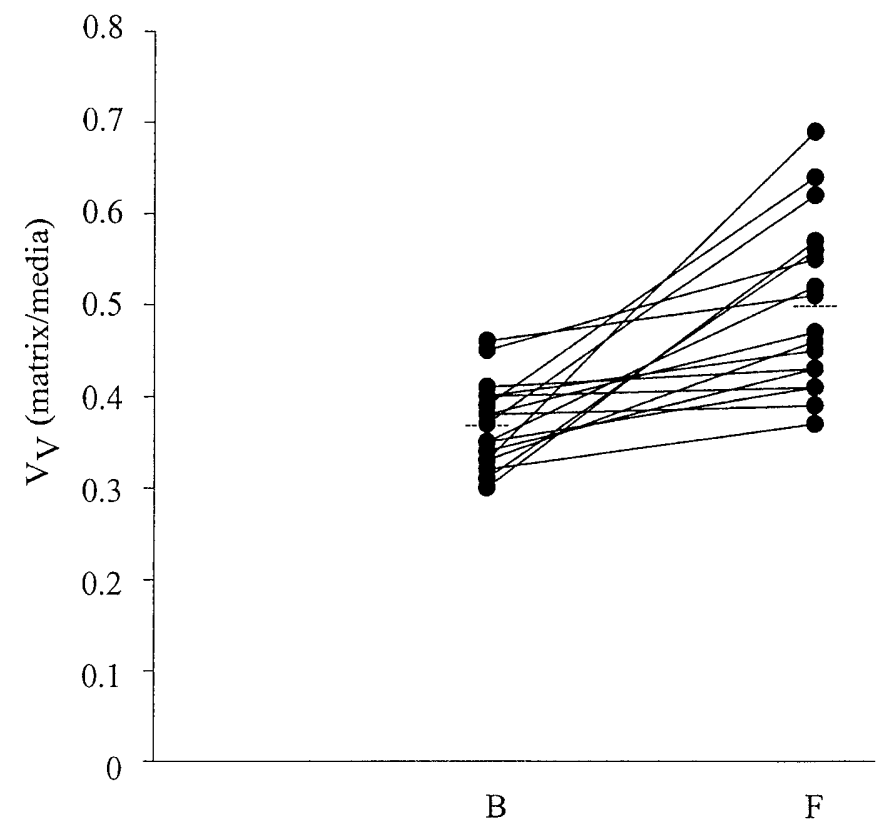

Fig. 5. Afferent arterioles: Matrix volume fraction in arteriolar media, $\mathrm{V}_{\mathrm{V}}$ (matrix/media) in afferent arterioles in individual patients. The increase from baseline to follow-up is highly significant $(p=0.0001)$

Structural data in baseline and follow-up biopsies. The ultrastructure of the extra-cellular material at some places showed fluffy appearance, and at other demarcated areas would appear electron-dense (Figs.3-4). Accumulations of vehicles and membranous structures also occurred (Fig.3). Some myomedial cells showed degenerative changes, e. g. marked vacuolisation (Fig. 3).

Figures 5 and 6 show data in individual patients of $\mathrm{V}_{\mathrm{V}}$ (matrix/media) in afferent (Fig.5) and efferent (Fig. 6) arterioles. The increase over the period is highly significant $(p=0.0001$ for the afferent arterioles, and $p=0.0004$ for the efferent arterioles).

Table 2 shows the calculated thickness, T, corresponding to the average thickness of the particular component if spread out in an even layer of the arteriolar circumference $[8,10]$, calculated for matrix, total media, endothelial cells and the corresponding estimate of luminal radius. The increase in matrix and total media thickness in both afferent and efferent arterioles is highly significant, whereas the endothelial height showed a significant decrease, and no change was found in the luminal radius.

As to the structural appearance of the extra-cellular material, the fluffy material, as a fraction of total matrix, decreased from baseline to 8 years in both afferent $(p=0.001)$ and efferent arterioles $(p=0.03)$. The fraction of electron dense material on the other hand increased, but only significant in afferent arterioles $(p=0.02)$. 


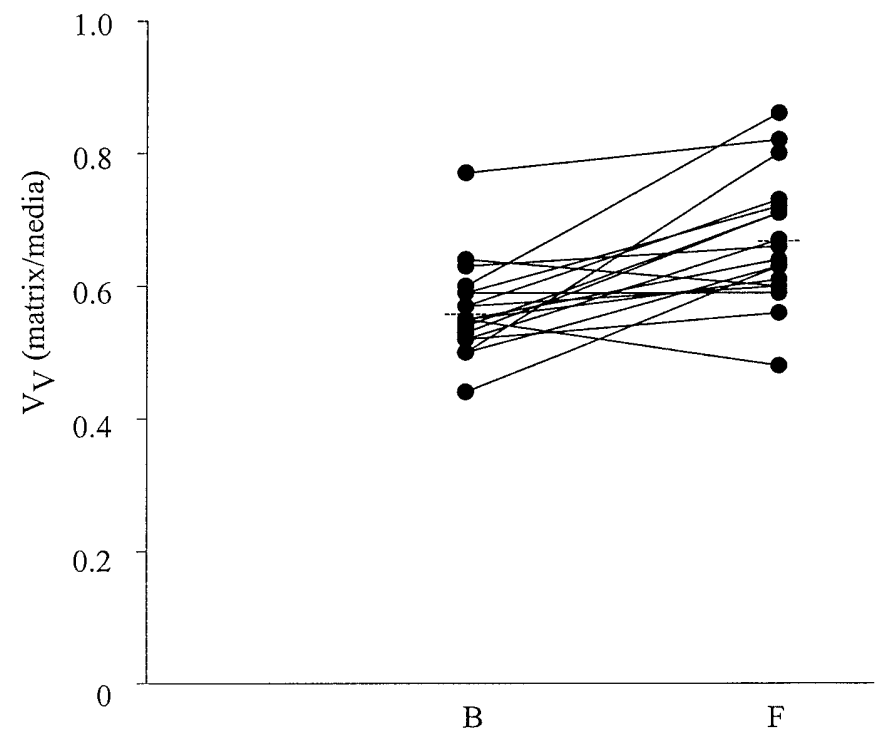

Fig. 6. Efferent arterioles: Volume fraction of matrix in media, $\mathrm{V}_{\mathrm{V}}$ (matrix/media) in efferent arterioles in baseline and followup biopsies $(p=0.0004)$

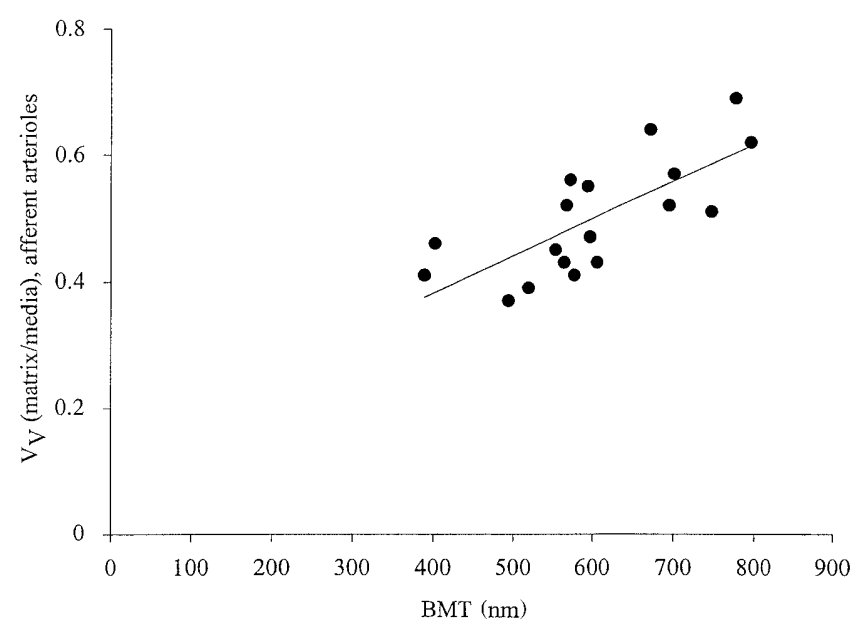

Fig. 7. Correlation between afferent $\mathrm{V}_{\mathrm{V}}$ (matrix/media) and glomerular basement membrane thickness in 8-years biopsies $(r=0.74, p=0.0005)$

Correlations among structural parameters: Arteriolar structure compared with glomerulopathy. Table 3 shows the correlation data for matrix-to-media volume fractions in afferent and efferent arterioles compared with the quantitative data on glomerulopathy. Clearly among the biopsies, those with most advanced glomerulopathy showed the most advanced alterations in the arteriolar walls. The correlations stand out most clearly for the afferent arterioles, as illustrated in Figure 7.

The increase in $\mathrm{V}_{\mathrm{V}}$ (matrix/media) from baseline to 8 years showed significant correlation with the concurrent increase in the glomerular basement membrane thickness (Table 4). A significant correlation was seen between the increase in afferent $\mathrm{V}_{\mathrm{V}}$ (matrix/
Table 2. Calculated thickness, $\mathrm{T}$, for arteriolar profiles without connection to the glomerular vascular pole. Means $(\mathrm{CV} \%=$ $\mathrm{SD} /$ mean $\%$ ). Students paired $t$ test

\begin{tabular}{llll}
\hline & $\begin{array}{l}\text { Baseline } \\
\text { biopsies }\end{array}$ & $\begin{array}{l}\text { 8-years } \\
\text { biopsies }\end{array}$ & $p$ \\
\hline Afferent arterioles, & 1.61 & 2.73 & $<10^{-4}$ \\
Mat-T $(\mu \mathrm{m})$ & $(13)$ & $(31)$ & \\
Efferent arterioles, & 1.43 & 2.10 & 0.0004 \\
Mat-T $(\mu \mathrm{m})$ & $(20)$ & $(37)$ & \\
Afferent arterioles, & 4.50 & 5.50 & 0.0002 \\
Media-T $(\mu \mathrm{m})$ & $(9)$ & $(17)$ & \\
Efferent arterioles, & 2.49 & 3.10 & 0.002 \\
Media-T $(\mu \mathrm{m})$ & $(12)$ & $(24)$ & \\
Afferent arterioles, & 1.00 & 0.75 & 0.05 \\
Endothelium-T $(\mu \mathrm{m})$ & $(46)$ & $(19)$ & \\
Efferent arterioles, & 1.04 & 0.78 & 0.0003 \\
Endothelium-T $(\mu \mathrm{m})$ & $(19)$ & $(19)$ & \\
Afferent arterioles, & 2.46 & 2.42 & $\mathrm{~ns}$ \\
Luminal radius $(\mu \mathrm{m})$ & $(29)$ & $(28)$ & \\
Efferent arterioles, & 2.55 & 2.73 & $\mathrm{~ns}$ \\
Luminal radius $(\mu \mathrm{m})$ & $(19)$ & $(14)$ & \\
\hline
\end{tabular}

Table 3. Correlations between arteriolar structure and parameters of diabetic glomerulopathy in the 8-years biopsies

\begin{tabular}{llllll}
\hline & \multicolumn{2}{l}{$\begin{array}{l}\mathrm{V}_{\mathrm{V}} \text { (matrix/media) } \\
\text { afferent arterioles }\end{array}$} & & \multicolumn{2}{l}{$\begin{array}{l}\mathrm{V}_{\mathrm{V}} \text { (matrix/media) } \\
\text { efferent arterioles }\end{array}$} \\
\cline { 2 - 3 } $\begin{array}{llllll}\text { Basement membrane } \\
\text { thickness }\end{array}$ & 0.74 & 0.0005 & & 0.58 & 0.01 \\
$\mathrm{~V}_{\mathrm{V}}$ (mes/glom) & 0.50 & 0.03 & & 0.45 & 0.06 \\
$\mathrm{~V}_{\mathrm{V}}$ (matrix/glom) & 0.69 & 0.001 & & 0.63 & 0.005 \\
Matrix star volume & 0.43 & 0.07 & & 0.36 & 0.15
\end{tabular}

Mesangial matrix star volume is a measure which increases with increasing size, convexity and confluence of the individual matrix segments (ref No. [11])

media) and the increase in the mesangial volume fraction in the afferent arterioles, whereas no significant correlation was seen concerning the efferent arterioles. Increases in $\mathrm{V}_{\mathrm{V}}$ (matrix/media) in afferent arterioles correlated with increases in $\mathrm{V}_{\mathrm{V}}$ (matrix/glom).

Arteriolar structure compared with other structural measures. Over the 8 years, increases in glomerular volume and the vascular pole area have been reported [12]. Whereas no correlation was found between glomerular volume and arteriolar structure in the 8years biopsies, there was a tendency, not statistically significant, to a positive correlation with the vascular pole area, $(r=0.44, p=0.065$ for afferent; $r=0.46$, $p=0.057$ for efferent arterioles).

Measurements of the volume of the juxtaglomerular apparatus (JGA) have also been completed (C Gulmann, unpublished). In the 8-year biopsies a significant correlation was seen between JGA volume 
Table 4. Correlations between changes in arteriolar structure compared with changes in parameters of diabetic glomerulopathy from baseline to 8-years biopsies

\begin{tabular}{|c|c|c|c|c|}
\hline & \multicolumn{2}{|c|}{$\begin{array}{l}\Delta \mathrm{V}_{\mathrm{V}}(\text { matrix/media }) \\
\text { afferent arterioles }\end{array}$} & \multicolumn{2}{|c|}{$\begin{array}{l}\Delta \mathrm{V}_{\mathrm{V}} \text { (matrix/media) } \\
\text { efferent arterioles }\end{array}$} \\
\hline & $r$ & $p$ & $R$ & $\mathrm{p}$ \\
\hline $\begin{array}{l}\Delta \text { Basement mem- } \\
\text { brane thickness }\end{array}$ & 0.63 & 0.005 & 0.71 & 0.001 \\
\hline$\Delta \mathrm{V}_{\mathrm{V}}($ mes/glom $)$ & 0.51 & 0.03 & 0.33 & 0.18 \\
\hline$\Delta \mathrm{V}_{\mathrm{V}}($ matrix/glom $)$ & 0.48 & 0.046 & 0.29 & 0.24 \\
\hline$\Delta$ Matrix star volume & 0.38 & 0.12 & 0.30 & 0.22 \\
\hline
\end{tabular}

and the arteriolar $\mathrm{V}_{\mathrm{V}}$ (matrix/media) $\quad(r=0.61$, $p=0.02$ for afferent arterioles, $r=0.75, p=0.0012$ for efferent arterioles).

Significant correlations were not observed between change in JGA volume and change in arteriolar structure.

The volume fraction of cortical interstitium did not correlate with the arteriolar measures.

In the 8-year biopsies but not in the baseline biopsies the correlation between matrix volume fraction in afferent and that in efferent arterioles was significant $(r=0.60, p=0.009)$.

Correlations with clinical variables. Neither age, diabetes duration nor the clinical blood pressure during the study period showed any correlations with arteriolar structure.

The value of AER (log transformed) at the final biopsy showed significant correlation with both afferent (Fig. 8) and efferent parameters $(r=0.49, p=0.04$ and $r=0.59, p=0.01$, respectively). The five patients who had normoalbuminuria at termination had lower arteriolar parameters (not significant), and these patients also had a significantly lower $\mathrm{HbA}_{1 \mathrm{C}}$ over the 8 years compared with the remaining cases $(p=0.02)$.

With GFR at follow-up, inverse correlation existed with both sets of arterioles $(r=-0.60 p=0.009$ for afferent, and $r=-0.61, p=0.007$ for efferent). Also the change in GFR over the study period showed an inverse correlation with arteriolar data at follow-up $(r=-0.57, p=0.01$ for afferent, $r=-0.51, p=0.03$ for efferent arterioles).

$\mathrm{HbA}_{1 \mathrm{C}}$ during the study period tended to correlate with arteriolar composition ( $p=0.07$ and $p=0.08$ for afferent and efferent arterioles, respectively).

The two subgroups of the series, one treated during the initial 21/2-3 years with conventional treatment, the other with intensified treatment, did not show significant differences in arteriolar parameters at the follow-up. Whereas the $\mathrm{HbA}_{1 \mathrm{C}}$ was significantly lower in the intensively treated group during the controlled trial, this difference did not hold through when the entire period of 8 years is considered.

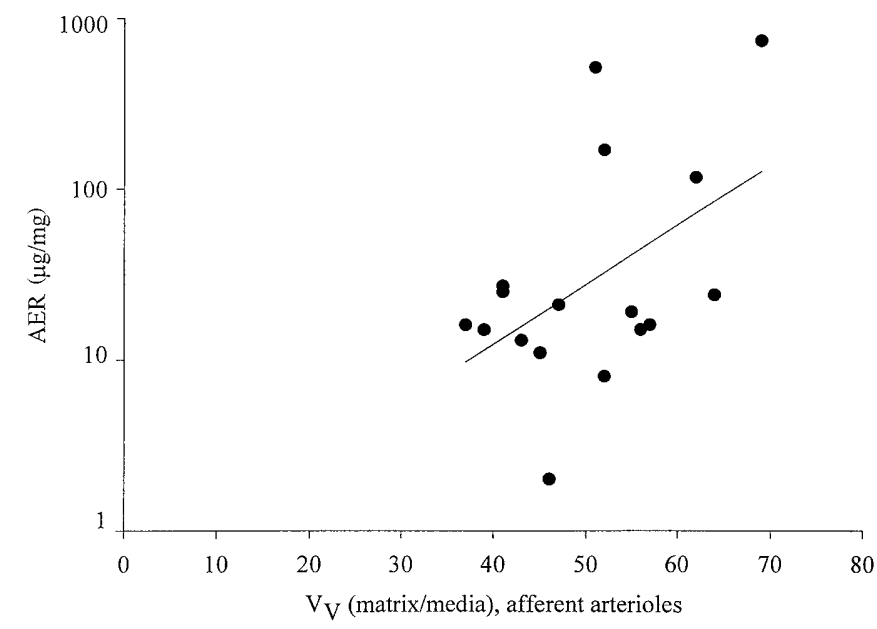

Fig. 8. Correlation between $\mathrm{V}_{\mathrm{V}}$ (matrix/media) and the albumin excretion rate on a log scale in the 8-years biopsies $(r=0.49, p=0.04)$

\section{Discussion}

In earlier semi-quantitative studies of arteriolar hyalinosis dealing with a wide range of renal function in Type I diabetic patients, an association with the clinical stage was observed $[9,13]$. Also in this series of diabetic patients with a duration of disease of about 20 years, covering a much narrower clinical window, we saw an inverse correlation with GFR and a positive correlation with AER. The five patients in the present series who had reverted to normoalbuminuria had lower arteriolar parameters. However, this small subset of patients was not statistically different from the remaining group.

The segments of arterioles which are completely devoid of myomedial cells probably do not respond to signals from intravascular pressure: The normal auto-regulation is lost. Thus, in situations where increases in pressure are involved, the glomerulus is not protected from the projection of the pressure into the glomerular tuft. This might well contribute to the increased leakiness of the glomerular capillaries, in particular if the filter is not of a completely normal composition as in the diabetic glomerulopathy. The arteriolar hyalinosis could thereby be part of the structural explanation of the higher albumin excretion [24]. Also, in situations with hypotension or during increased sympathetic tone or other vasoconstrictive stimulation such segments are probably not able to dilate, which could then lead to damage due to hypoperfusion and hypofiltration.

The inverse relation between GFR and arteriolar hyalinosis in the 8-years biopsies cannot be immediately explained. It could in fact be related to the association between arteriolar and glomerular lesions. More advanced glomerulopathy was shown in this group of patients to correlate with lower GFR (Bangstad et al, unpublished). 
We were not able to find any association between the arteriolar changes and the glomerular occlusion. However, the latter phenomenon is quite infrequent in the series of patients who are at a stage of incipient nephropathy [12]. Arteriolar hyalinisation has been implied in the process of glomerular occlusion [9]. Other studies of very large series, not dealing with diabetic patients in particular, suggested a much clearer influence of the subintimal lesions of the renal arteries [15]. Impressions from studies of the serial sections indicate that subintimal fibrosis at the site of departure of an afferent arteriole could be decisive (personal observation). These changes are definitely also present in microalbuminuric diabetic patients but they are not easily accessible to quantitative measures in renal biopsy material.

Association between the severity of diabetic glomerulopathy and the arteriolar changes as found in the present series was also reported in semi-quantitative studies $[9,13]$ and in the previous quantitative study [8]. The diabetic glomerulopathy as well as the arteriolopathy are first and foremost characterised by the accumulation of extra-cellular material. In the arterioles, a heterogeneous appearance of the material was often noted with fluffy areas or electron dense segments or both. Also the mesangial matrix in advanced glomerulopathy shows a heterogeneous ultrastructural appearance [16]. The decrease in fluffy material over the 8 years could represent a change of the biochemical composition of the extra-cellular material. The clearly demarcated electron dense areas, on the other hand, increased. It seems likely that they, to some extent, represent accumulations of extravasated plasma proteins, which could increase along with increasing albumin excretion $[3,17]$.

In the small series we could demonstrate not only an association between the severity of glomerulopathy and arteriolopathy in the 8-years biopsies, but also the change from baseline to follow-up biopsy, which showed a parallel course at the two sites. It seems reasonable to assume that the underlying mechanism for all of these changes is a metabolically determined increase in matrix production. This is strongly supported by in vitro studies showing increased production by cultured mesangial cells if grown in high glucose [18]. Similarly, myomedial cells isolated from larger arteries (aorta) increase matrix production when cultured in serum from diabetic patients [19]. The same mechanism could very well be at operation in the arterioles.

It has recently been shown in in vitro experiments that the application of stretch to mesangial cells increases the matrix production, and increased glomerular pressure is thought to play a role in the development of glomerulopathy [20, 21]. Experiments using arteriolar cells have probably not been done but it could well be that a similar stretch effect has an influence on the arteriolar wall composition. The myome- dial cells are in fact analogous with the mesangial cells. A slight increase in systemic blood pressure, as detectable by 24 -h measurements in early microalbuminuria [22] could therefore also contribute to the arteriolar lesions observed in this study, even if elevation of the clinically measured blood pressure did not occur.

Interestingly, in this context, in essential hypertension the arterioles respond, at least initially, with hypertrophy of the myomedial cells $[5,23]$. In this diabetic series, we rather saw degenerative changes and the disappearance of these cells.

In contrast with non-diabetic individuals, the efferent arterioles are also affected in the diabetic state. It is not clear whether the efferent arterioles in non-diabetic individuals are not carrying a high pressure, whereas pressure is projected all the way through the glomerular tuft to the efferent arteriole in diabetic patients. We found in earlier studies that the area of the vascular pole region increases in diabetic patients and it increased over the 8-year period in the present material. Furthermore, the average area of the efferent arteriole in the vascular pole increased from baseline to 8 years (C Gulmann, unpublished).

The arteriolopathy is markedly focal. The cause for this focal distribution is not known. It indicates that other factors together with the metabolic environment are at operation at certain segments. The focal appearance is in fact not different from that found in a variety of vasculopathies.

If the changes of the juxtaglomerular arterioles could influence the glomerular tuft to which they belong, the focal nature of the arteriolopathy could be an important contributor to increased heterogeneity of the glomerulopathy in more advanced cases of diabetic nephropathy.

In conclusion, we found that the arteriolar accumulation of matrix is a progressing process in young Type I diabetic patients with microalbuminuria. The concordance between the severity of glomerulopathy and arteriolopathy could indicate common pathogenetic mechanisms or interaction between the lesions at the two locations or both. The arteriolopathy in concert with the glomerulopathy are most likely the determinants of the development of clinical diabetic nephropathy.

Acknowledgements. Expert technical assistance from B. Iversen, L. Lysgaard and B. Saugbjerg is gratefully acknowledged, as is the typing of the manuscript by K. $\varnothing$. Kristensen, MA. This study was supported by grants from Aarhus University Foundation, the Danish Diabetes Association, The Danish Medical Research Council, The Juvenile Diabetes Foundation International no. 190592, Novo Nordic Foundation, the Norwegian Diabetes Association. 


\section{References}

1. Thomsen AC (1965) The Kidney in Diabetes Mellitus. Munksgaard, Copenhagen, Denmark

2. Olsen S (2000) Light microscopy of diabetic glomerulopathy: The classic lesions. In: Mogensen CE (ed) The Kidney and Hypertension in Diabetes Mellitus, $5^{\text {th }}$ edn. Kluwer Academic Publishers, Boston, pp 201-210

3. Parving HH (1975) Microvascular permeability to plasma proteins in hypertension and diabetes mellitus in man - on the pathogenesis of hypertensive and diabetic microangiopathy. Dan Med Bull 22: 217-233

4. Tracy RE, Strong JP, Newman WP, Malcom GT, Oalmann MC, Guzman MA (1996) Renovasculopathies of nephrosclerosis in relation to atherosclerosis at ages 25 to 54 years. Kidney Int 49: 564-570

5. Berk BC, Alexander RW (2000) Biology of the Vascular Wall in Hypertension. In: Brenner BM (ed) The Kidney, $6^{\text {th }}$ edn, W.B.Saunders Company, Philadelphia, pp 2049-2070

6. Mauer SM, Barbose J, Vernier RL et al. (1976) Development of diabetic vascular lesions in normal kidneys transplanted into patients with diabetes mellitus. N Engl J Med 295: 916-920

7. Ellis EN, Warady BA, Wood EG et al. (1997) Renal structural-functional relationships in early diabetes mellitus. Pediatr Nephrol 11: 584-591

8. Østerby R, Bangstad H-J, Nyberg G, Walker JD, Viberti G (1995) A quantitative ultrastructural study of juxtaglomerular arterioles in IDDM patients with micro- and normoalbuminuria. Diabetologia 38: 1320-1327

9. Harris RD, Steffes MW, Bilous RW, Sutherland DE, Mauer SM (1991) Global glomerular sclerosis and glomerular arteriolar hyalinosis in insulin dependent diabetes. Kidney Int 40: 107-114

10. Gulmann C, Rudberg S, Østerby R (1999) Renal arterioles in Type I diabetic patients with microalbuminuria - before and after treatment with either an ACE inhibitor or a beta-blocker. Virchows Arch 434: 523-528

11. Bangstad H-J, Østerby R, Dahl-Jørgensen K et al. (1993) Early glomerulopathy is present in young, Type I (insulindependent) diabetic patients with microalbuminuria. Diabetologia 36: 523-529

12. Østerby R, Hartmann A, Nyengaard JR, Bangstad H-J (2002) Development of renal structural lesions in Type 1 diabetic patients with microalbuminuria - Observations by light microscopy in 8-years' follow-up biopsies. Virchows Arch 440: 94-101

13. Lane PH, Steffes MW, Fioretto P, Mauer M (1993) Renal interstitial expansion in insulin-dependent diabetes mellitus. Kidney Int 43: 661-667

14. Østerby R (1995) Microalbuminuria in diabetes mellitus is there a structural basis? Nephrol Dial Transplant 10: 12-14

15. Tracy RE (1999) The heterogeneity of vascular findings in the kidneys of patients with benign essential hypertension. Nephrol Dial Transplant 14: 1634-1639

16. Østerby R (1974) Early phases in the development of diabetic glomerulopathy. Acta Med Scand Suppl 574: 3-82

17. Westberg NG, Michael AF (1972) Immunohistopathology of diabetic glomerulosclerosis. Diabetes 21: 163-174

18. Ayo SH, Radnik RA, Glass WF et al. (1991) Increased extracellular matrix synthesis and mRNA in mesangial cells grown in high-glucose medium. Am J Physiol 260: F185F191

19. Ledet T, Vuust J (1980) Arterial procollagen type I, and type III, and fibronectin: effects of diabetic serum, glucose, insulin, ketone, and growth hormone studied on rabbit aortic myomedial cell cultures. Diabetes 29: 964-970

20. Cortes P, Zhao X, Riser BL, Narins RG (1997) Role of glomerular mechanical strain in the pathogenesis of diabetic nephropathy. Kidney Int 51: 57-68

21. Gruden G, Zonca S, Hayward A et al. (2000) Mechanical stretch-induced firbonectin and transforming growth factor-beta1 production in human mesangial cells is p38 mitogen-activated protein kinase-dependent. Diabetes 49: 655-661

22. Poulsen PL, Hansen KW, Mogensen CE (1994) Ambulatory blood pressure in the transition from normo- to microalbuminuria. A longitudinal study in IDDM patients. Diabetes 43: $1248-1253$

23. Korsgaard N, Mulvany MJ (1988) Cellular hypertrophy in mesenteric resistance vessels from renal hypertensive rats. Hypertension 12: 162-167

24. Mogensen CE (1999) Microalbuminuria, blood pressure and diabetic renal disease: origin and development of ideas. Diabetologia 42: 263-285

25. Parving HH, Østerby R, Ritz E (2000) Diabetic nephropathy. In: Brenner BM (ed) The Kidney, $6^{\text {th }}$ edn, WB Saunders Co, Philadelphia, pp 1731-1773 